\title{
Growth and Optical Characterisation of Lithium Fluoride Films for Proton Beam Detectors
}

\author{
Maria Aurora Vincenti ${ }^{1}$, Mauro Leoncini ${ }^{2}$, Stefano Libera ${ }^{1}$, Enrico Nichelatti ${ }^{3}$, \\ Massimo Piccinini ${ }^{1}$, Alessandro Ampollini ${ }^{1}$, Luigi Picardi ${ }^{1}$, Concetta Ronsivalle ${ }^{1}$, \\ Alessandro Rufoloni ${ }^{1}$ and Rosa Maria Montereali ${ }^{1}$ \\ ${ }^{1}$ Fusion and Technologies for Nuclear Safety and Security Dept., ENEA C.R. Frascati, Frascati \\ (Rome), Italy \\ ${ }^{2}$ CNR NANOTEC, Campus Ecotekne, Lecce, Italy \\ ${ }^{3}$ Fusion and Technologies for Nuclear Safety and Security Dept., ENEA C.R. Casaccia, \\ S. Maria di Galeria (Rome), Italy \\ *aurora.vincenti@enea.it
}

Keywords: Lithium Fluoride, Thin Films, Colour Centres, Photoluminescence, Radiation Detectors

\begin{abstract}
Polycrystalline LiF thin films were grown by thermal evaporation on glass, fused silica (Suprasil ${ }^{\circ}$ ) and $\mathrm{Si}(100)$ substrates in controlled conditions. Starting from the measured specular reflectance and direct transmittance spectra, some physical parameters of the LiF films grown on fused silica substrate were determined by using a best-fit procedure. The LiF films grown on glass and $\mathrm{Si}(100)$ substrates were irradiated by proton beams of $27 \mathrm{MeV}$ nominal energy produced by a pre-clinical linear accelerator at several doses in the range between $4.2 \times 10^{3}$ and $1.7 \times 10^{5}$ Gy. Substrate-enhanced photoluminescence intensity was observed in coloured LiF films grown on Si substrates with respect to LiF films deposited on glass in the same deposition run. This behaviour is mainly ascribed to the reflective properties of the Si substrate in the visible spectral range, where the absorption and emission bands of $\mathrm{F}_{2}$ and $\mathrm{F}_{3}{ }^{+}$ CCs are located, although other complex effects due to the polycrystalline nature of the films cannot be excluded. Further systematic studies are under way.
\end{abstract}

\section{Introduction}

Optical and chemical-physical properties of lithium fluoride (LiF) and its sensitivity to ionising radiations make this material promising for several applications ranging from photonics [1] to dosimetry [2]. LiF crystals are hard and almost non-hygroscopic, two precious properties for applications. $\mathrm{LiF}$ band gap is greater than $14 \mathrm{eV}$, so it is optically transparent from $120 \mathrm{~nm}$ to 7 $\mu \mathrm{m}$ and for this reason it is a widely used window material, in particular in the UV spectral region. Irradiations by ionising radiations, such as X-rays, $\gamma$-rays, protons, neutrons, electrons, etc., induces the formation of electronic defects, known as colour centres (CCs), which are stable at room temperature (RT). Among them, the aggregate $\mathrm{F}_{2}$ and $\mathrm{F}_{3}{ }^{+} \mathrm{CCs}$ (two electrons bound to two and three close anion vacancies, respectively) have almost overlapped absorption bands peaked at wavelengths of 444 and $448 \mathrm{~nm}$, respectively; these together form the $\mathrm{M}$ absorption band, located in the blue spectral region [3]. Under optical excitation in the $\mathrm{M}$ band, the $\mathrm{F}_{2}$ and $\mathrm{F}_{3}{ }^{+}$CCs simultaneously emit broad Stokes-shifted photoluminescence (PL) bands peaked at 678 and $541 \mathrm{~nm}$, respectively.

In recent years, the area of growth and characterisation of $\mathrm{LiF}$ films for radiation imaging detectors has seen a growing interest. LiF-film-based detectors can be grown by thermal 
evaporation on different substrates in controlled conditions, tailoring the appropriate geometry, size and thickness. These detectors are multi-purpose, allowing to detect X-rays, protons, neutrons, electrons, etc.. They are insensitive to the ambient light and they do not need any development process after the exposure. Their reading technique is based on the optical detection of the PL signal emitted by radiation-induced $\mathrm{F}_{2}$ and $\mathrm{F}_{3}{ }^{+}$CCs. LiF films grown by thermal evaporation were proposed and tested as novel extreme-ultraviolet $(20<\mathrm{hv}<300 \mathrm{eV})$ and soft X-rays $(0.3<\mathrm{hv}<3 \mathrm{keV})$ imaging detectors [4,5] based on $\mathrm{F}_{2}$ and $\mathrm{F}_{3}{ }^{+} \mathrm{PL}$, as well as nuclear sensors for neutrons [6], for the characterisation of coherent X-ray sources [7] and advanced diagnostics of proton beams $[8,9]$.

In this work preliminary experimental results about the enhancement of the emission properties of LiF-film-based detectors irradiated by $27 \mathrm{MeV}$ proton beams at doses in the range between $4.2 \times 10^{3}$ and $1.7 \times 10^{5}$ Gy are presented and discussed.

\section{Materials and methods}

Polycrystalline LiF films, of nominal thicknesses 660, 1160 and $1450 \mathrm{~nm}$, were grown by thermal evaporation on glass, fused silica and $\mathrm{Si}(100)$ substrates at ENEA C.R. Frascati. The deposition processes were performed in a steel vacuum chamber at a pressure below $1 \mathrm{mPa}$. After substrate cleaning, performed by using detergents in ultrasonic baths, the substrates were mounted on a rotating sample-holder placed above a tantalum crucible at a distance of $22 \mathrm{~cm}$ from it. During the deposition process, the substrate temperature was kept constant at $300{ }^{\circ} \mathrm{C}$ by means of four infrared halogen lamps controlled by the WEST 6400 thermoregulator and a calibrated thermocouple positioned inside the vacuum chamber. LiF powder (Merck Suprapur, $99.99 \%$ pure), placed in the crucible, was heated at about $850{ }^{\circ} \mathrm{C}$ by Joule effect. The deposition rate and the film thickness were monitored in situ by an INFICON quartz oscillator. All the deposition processes were performed keeping the deposition rate at a value of $1 \mathrm{~nm} / \mathrm{s}$.

After the growth, the thickness of the deposited LiF films was measured by using a Tencor P10 stylus profilometer.

The morphological analysis was performed by means of a PARK System Atomic Force Microscopy (AFM), model XE-150, operating in air in non-contact mode.

The LiF films were irradiated in air at RT by proton beams at nominal energy of $27 \mathrm{MeV}$, produced by the injector of the pre-clinical linear accelerator TOP-IMPLART (Oncological Therapy with Protons-Intensity Modulated Proton Linear Accelerator for RadioTherapy) under development at ENEA C.R. Frascati [10]. The irradiations were performed placing the LiF samples perpendicularly to the proton beam at a distance of $10 \mathrm{~mm}$ from the $50 \mu \mathrm{m}$ thick kapton window, which constitutes the exit of the machine beamline. The average beam current was 27.4 $\mu \mathrm{A}$ with a pulse charge of $77 \mathrm{pC} /$ pulse and a repetition rate of $10 \mathrm{~Hz}$. The irradiations were performed at several doses in the range between $4.2 \times 10^{3}$ and $1.7 \times 10^{5} \mathrm{~Gy}$. According to the simulations made with SRIM (The Stopping and Range of Ions in Matter) software [11], the linear energy transfer of protons can be considered as constant through the limited thickness of $\mathrm{LiF}$ films and equal to $\sim 4.5 \mathrm{keV} / \mu \mathrm{m}$. The $27 \mathrm{MeV}$ proton range within $\mathrm{LiF}$ is about $3.5 \mathrm{~mm}$, much larger than the film thicknesses, therefore only a very small fraction of the total proton energy is released within the films.

PL and photoluminescence-excitation (PLE) spectra were collected at RT with a Horiba Scientific Fluorolog-3 spectrofluorimeter Model FL3-11, equipped with a $450 \mathrm{~W}$ xenon lamp, automatic slits, single-grating excitation and emission spectrometers, and a Hamamatsu R928 photomultiplier, adopting a front-face detection geometry. 


\section{Results and discussion}

The main deposition parameters, such substrate material, substrate temperature $\left(T_{s}\right)$, deposition rate, film thickness, together with the method of substrate cleaning before coating, influence the film structural, morphological and optical properties, and the adhesion of the film to the substrate. When the substrate temperature is $T_{s}=300{ }^{\circ} \mathrm{C}$, the $T_{s} / T_{m}$ ratio, where $T_{m}$ is the melting temperature of the film material and both temperatures are expressed in Kelvin, is greater than 0.5, which corresponds to Zone III of the Structure Zone Model [12]. In these experimental conditions, the film growth is characterized by bulk diffusion of admolecules, with activation energies above $0.3 \mathrm{eV}$, resulting in a rough equiaxed grained real structure of the film. Figure 1 shows the 3D AFM images over an area of $(5 \times 5) \mu \mathrm{m}^{2}$ of two LiF films, of nominal thickness 660 $\mathrm{nm}$, grown on glass and $\mathrm{Si}(100)$ substrates in the same deposition run. The measured values of the Root Mean Square (RMS) roughness are about $9.6 \mathrm{~nm}$ for the LiF film grown on glass substrate and $11.0 \mathrm{~nm}$ for the LiF film deposited on $\mathrm{Si}(100)$ substrate.
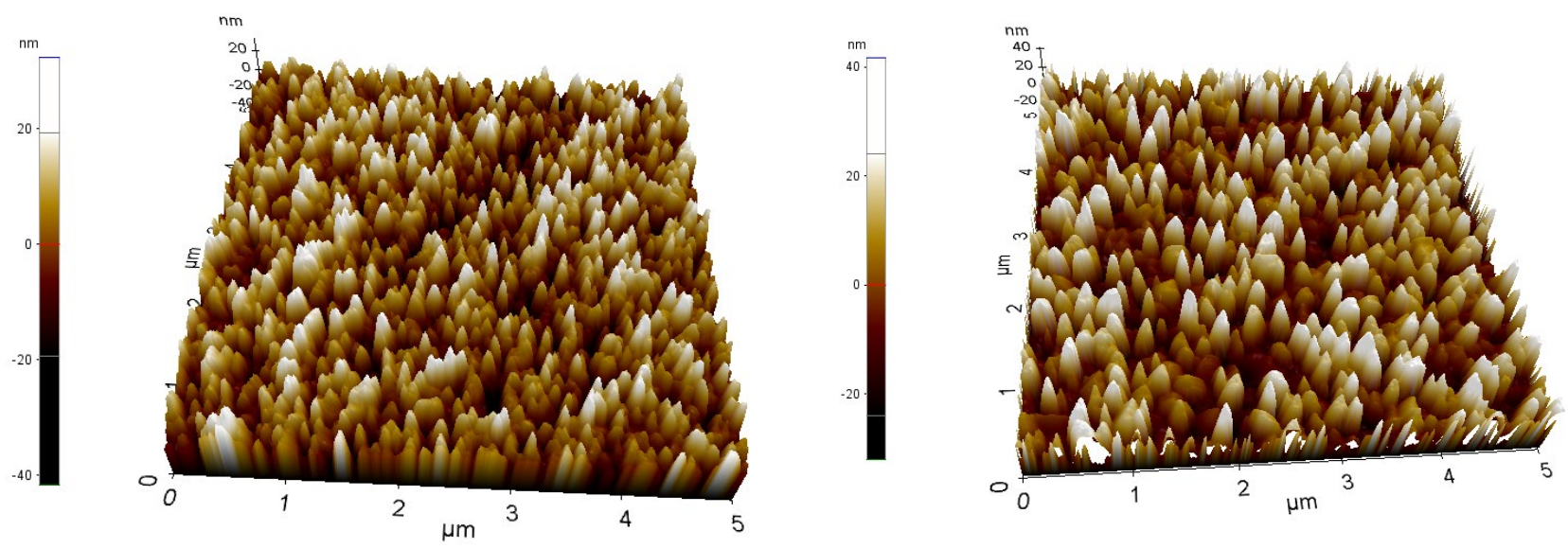

Fig. 1. 3D AFM images over an area of $(5 \times 5) \mu \mathrm{m}^{2}$ of two LiF films, of thickness $\sim 660 \mathrm{~nm}$, thermally-evaporated in the same deposition run on glass, left, and Si(100), right, substrates.

Optical and physical properties of the LiF films grown on Suprasil ${ }^{\circledR}$ were studied by analysing absolute specular reflectance and direct transmittance spectra measured in the 190 $\div 1600 \mathrm{~nm}$ wavelength range by using a Perkin-Elmer Lambda 900 spectrophotometer. By best fitting the experimental spectra with the film model introduced in [13,14], several features of the layer could be quantitatively estimated. As an example, Fig. 2 shows the measured specular reflectance and direct transmittance spectra, $R$ and $T$, of a LiF film of nominal thickness $660 \mathrm{~nm}$, together with their best fitting curves. The $R$ and $T$ spectra of a bare silica substrate, twin of the one on which the LiF film was evaporated, were also measured and are shown in Fig. 2 too. From the best fit procedure an average thickness of $(625 \pm 5) \mathrm{nm}$, a surface rootmean-square roughness of $(16.3 \pm 0.2) \mathrm{nm}$, a deviation from parallelism of the film faces of $(1.9 \pm 0.3) \%$ and a linear inhomogeneity along the growth axis of $(-1.5 \pm 0.4) \%$ were obtained.

The analysis allowed deriving the spectral dispersions of the film material refractive index and extinction coefficient as well, which are plotted in Fig. 3. 


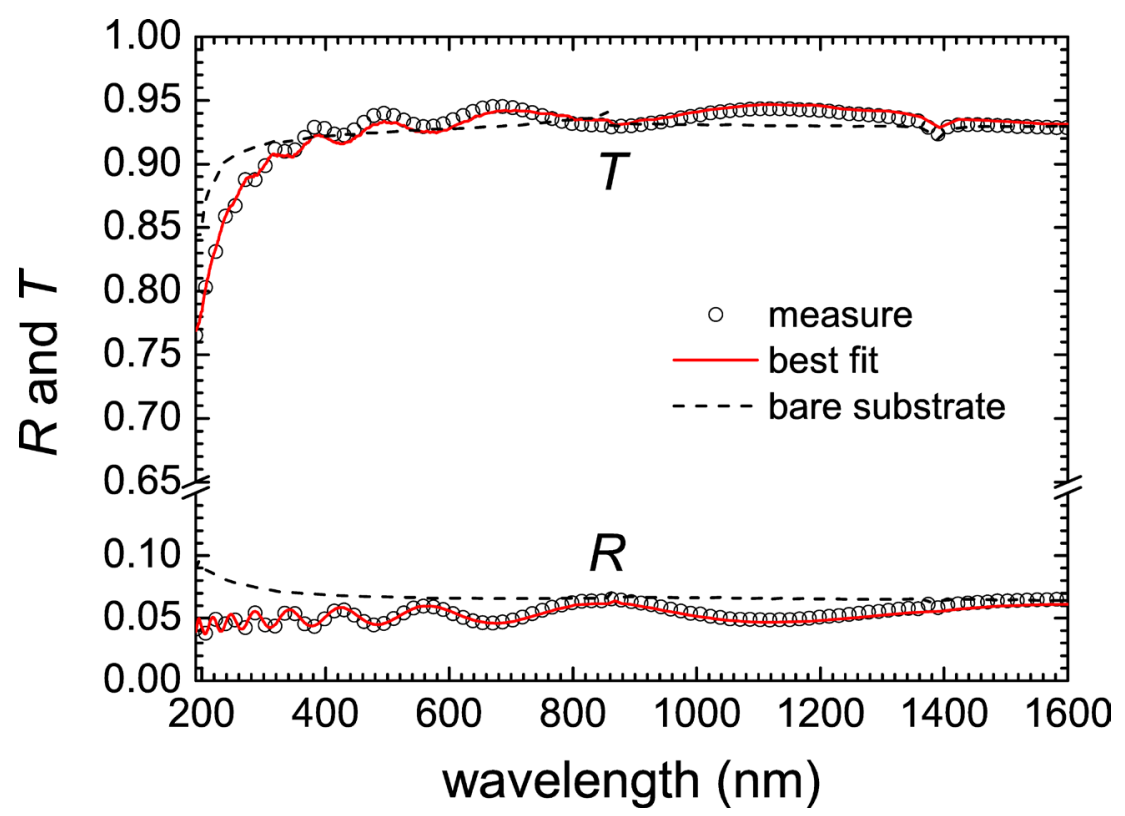

Fig. 2. Specular reflectance, $R$, and direct transmittance, $T$, spectra of the examined LiF film on silica substrate as measured at a Perkin-EImer Lambda 900 spectrophotometer and corresponding best-fitting theoretical spectra. The measured reflectance and transmittance of the bare silica substrate are also shown.

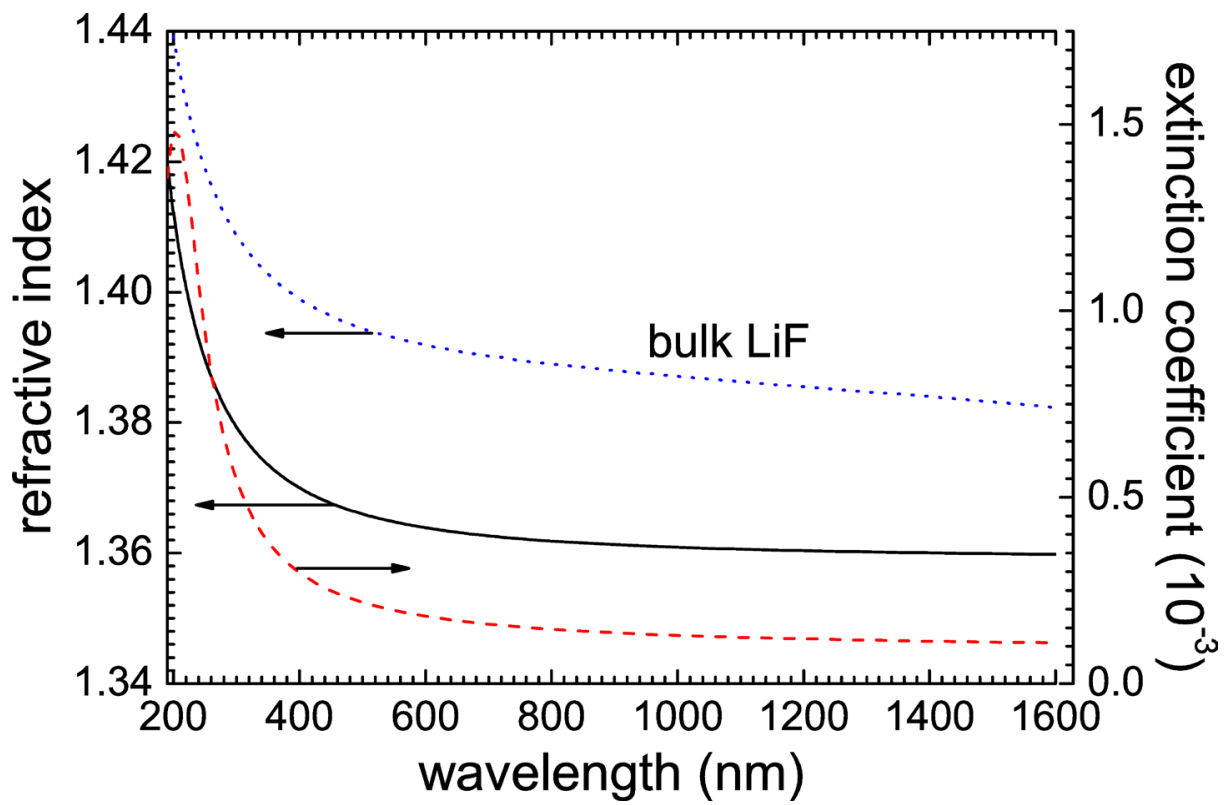

Fig. 3. Spectral dispersions of the LiF film refractive index (left scale) and extinction coefficient (right scale). The refractive index of bulk LiF [15] is also shown for comparison. 
By comparing the refractive-index dispersion with tabulated spectral data of bulk LiF [15], also plotted in Fig. 3, another important feature of the film material was estimated: the packing density. This quantity represents the fraction of volume in the layer which is effectively occupied by $\mathrm{LiF}$, the remaining fraction very likely consisting of air voids. Under the assumption that the sizes of such inhomogeneities are much smaller than the wavelengths of the incoming radiation, so that they are not sensed by it, this LiF-voids mixture can be approximately considered as a homogenous material whose refractive index can be evaluated by applying a suitable effectivemedium model [16]. In our case, application of the Maxwell Garnett equation [16] allowed finding the packing density value that gives the best superposition of the refractive-index spectral dispersion of a bulk-LiF-voids mixture with that obtained for the examined film; the result was an estimated packing density of about 93\%. Directly related to the packing density is the mass density of the film material: as a matter of fact, the latter should reasonably be equal to a weighted average of the mass densities of bulk LiF and air, the corresponding weights being equal to $93 \%$ and $7 \%$, respectively, for the examined sample. Assuming a bulk LiF mass density of $2.635 \mathrm{~g} / \mathrm{cm}^{3}$ [17], a mass density of about $2.451 \mathrm{~g} / \mathrm{cm}^{3}$ was estimated for the LiF layer.

LiF films irradiated by $27 \mathrm{MeV}$ proton beams get uniformly coloured along their thickness [8]. Figure 4 (left) shows the PL spectra of the LiF films, of the three different thicknesses ( 660, 1165 and $1450 \mathrm{~nm}$ ), grown on glass and $\mathrm{Si}(100)$ substrates in the same deposition run, irradiated by $27 \mathrm{MeV}$ proton beams at the highest dose of $1.7 \times 10^{5} \mathrm{~Gy}$, optically pumped at a wavelength of $445 \mathrm{~nm}$, which simultaneously excite the PL of $\mathrm{F}_{2}$ and $\mathrm{F}_{3}{ }^{+}$CCs. Each PL spectrum consists of the superposition of two broad emission bands due to these two radiation-induced defects, peaked at the wavelengths of 678 and $541 \mathrm{~nm}$, respectively. For each PL spectrum, the partial contributions of $\mathrm{F}_{2}$ and $\mathrm{F}_{3}{ }^{+} \mathrm{CCs}$ can be decoupled by using a best fit procedure with two Gaussian bands with fixed peak positions and widths, in agreement with literature available data [18]. Figure 4 (right) reports, as an example, the PL spectrum of the LiF film grown on glass substrate, thickness $1165 \mathrm{~nm}$, together with such a best fit; the partial Gaussian band contributions due to $\mathrm{F}_{2}$ and $\mathrm{F}_{3}{ }^{+}$defects are also reported.

The PL signal intensity, which depends on the number of radiation-induced CCs stored in $\mathrm{LiF}$, increases with the film thickness both for LiF films grown on glass and $\mathrm{Si}(100)$ substrates. It also increases with the irradiation dose [8,9], although this behaviour is not discussed here. For LiF films grown on glass and $\mathrm{Si}(100)$ substrates in the same deposition run, the PL signal of the $\mathrm{LiF}$ grown on $\mathrm{Si}(100)$ results higher than that of the $\mathrm{LiF}$ film grown on glass, for all the three thicknesses. The main reason of the observed PL enhancement is the high reflectivity of silicon in the visible spectral range, where the absorption and emission bands of $\mathrm{F}_{2}$ and $\mathrm{F}_{3}{ }^{+} \mathrm{CCs}$ are located. The reflective substrate is able to redirect a large fraction of $\mathrm{F}_{2}$ and $\mathrm{F}_{3}{ }^{+} \mathrm{PL}$ towards the detection system, that otherwise, as in the case of glass substrate, gets almost completely lost. Moreover, this reflected signal can constructively interfere with the straightly emitted one for all the orders of multireflection and at all the emission angles for suitable ratios of the film thickness to the wavelength. Indeed, the planar structure constituted by the coloured LiF film over the reflective substrate is an elementary half microcavity $[19,20]$.

PLE measurements are an effective method to investigate the absorption spectral features of $\mathrm{F}_{2}$ and $\mathrm{F}_{3}{ }^{+}$defects stored in LiF films grown on glass and Si substrates. In the case of LiF films grown on glass substrate, optical absorption spectra do not allow to identify the presence of radiation-induced $\mathrm{F}_{2}$ and $\mathrm{F}_{3}{ }^{+} \mathrm{CCs}$ because of the interference fringes due to the non-negligible refractive index difference between film and substrate. For LiF films grown on $\mathrm{Si}$, moreover, this investigation is precluded by the substrate opacity. 

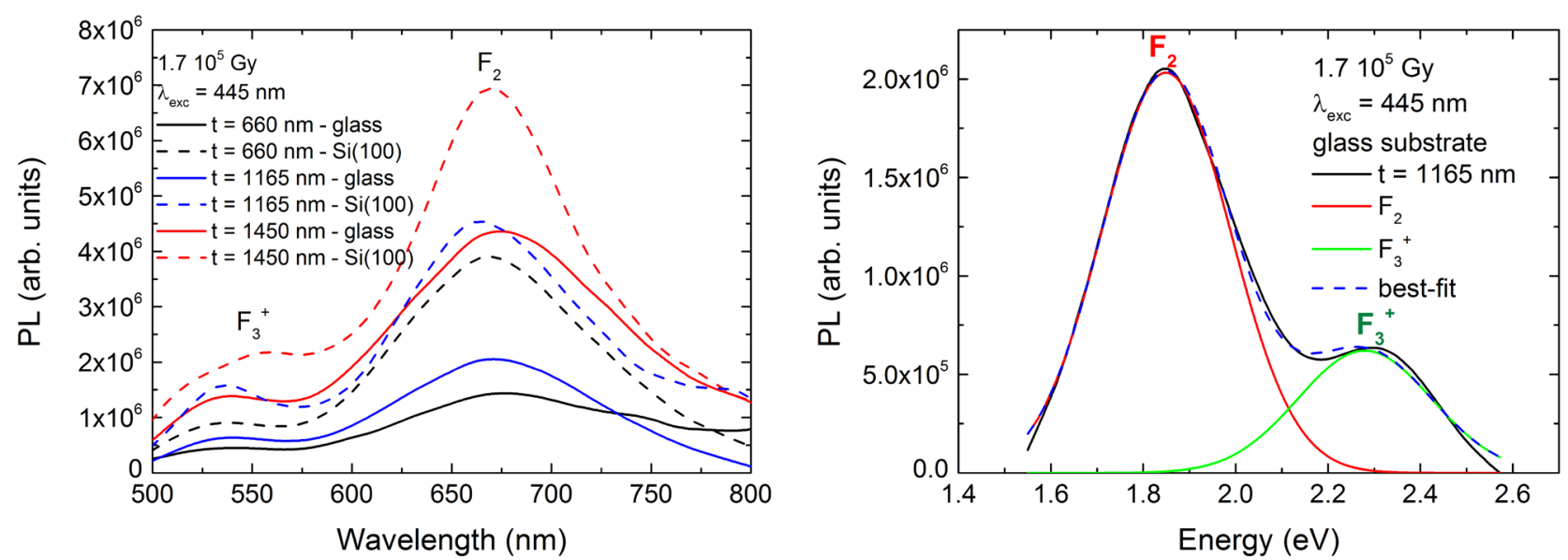

Fig. 4. (left) PL spectra of the LiF films thermally-evaporated on glass and on Si(100) substrates, of three thicknesses from 660 to $1450 \mathrm{~nm}$, irradiated by $27 \mathrm{MeV}$ proton beams at the dose of $1.7 \times 10^{5} \mathrm{~Gy}$, measured at the excitation wavelength of $445 \mathrm{~nm}$. (right) PL spectrum (solid black line) and best-fit (dashed blue line) of the LiF film grown on glass substrate, thickness $\sim 1165 \mathrm{~nm}$, irradiated by a $27 \mathrm{MeV}$ proton beam at a dose of $1.7 \times 10^{5} \mathrm{~Gy}$. The partial Gaussian band contributions due to $\mathrm{F}_{2}$ (solid red line) and $\mathrm{F}_{3}{ }^{+}$(solid green line) CCs obtained by best-fit, are also reported.

Figure 5 shows the PLE spectra of the LiF films grown on glass and Si substrates, nominal thickness $660 \mathrm{~nm}$, irradiated at $1.710^{5} \mathrm{~Gy}$, collected at the emission wavelength of 541 and 678 $\mathrm{nm}$. The blue vertical lines indicate the wavelength used to excite the PL spectra shown in Fig. 4.
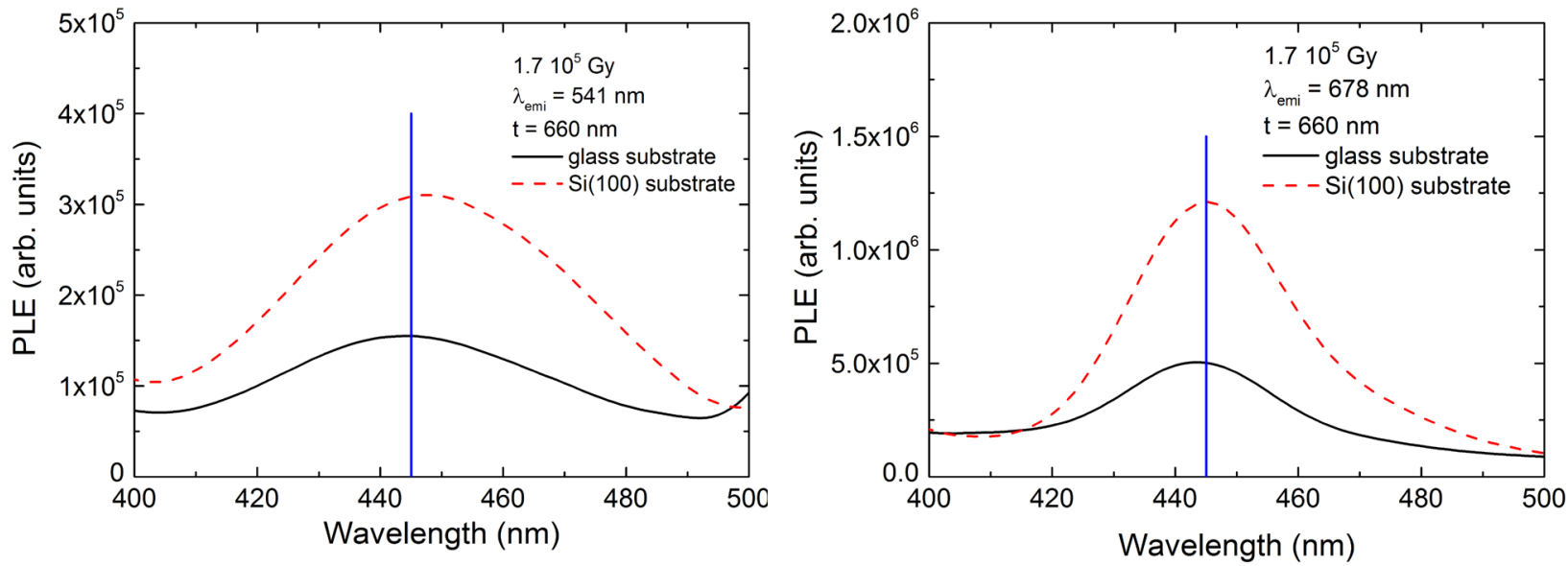

Fig. 5. (left) PLE spectra of the LiF films thermally-evaporated on glass and on Si(100) substrates, thickness $\sim 660 \mathrm{~nm}$, irradiated by $27 \mathrm{MeV}$ proton beams at a dose of $1.7 \times 10^{5} \mathrm{~Gy}$, acquired at the emission wavelength of $541 \mathrm{~nm}$ (contribution of $\mathrm{F}_{3}{ }^{+} \mathrm{CCs}$ ), left, and $678 \mathrm{~nm}$

(contribution of $F_{2} C C s$ ), right. The vertical blue line indicates the wavelength used to simultaneously excite the PL spectra of the samples shown in Fig. 4. 
For both LiF films grown on glass and $\mathrm{Si}(100)$ substrate, the $\mathrm{F}_{3}{ }^{+} \mathrm{PLE}$ band is broader than the $\mathrm{F}_{2}$ one and its peak position is located at longer wavelength, in agreement with the absorption spectral features of these radiation-induced defects known from literature [18].

The PLE signal collected at both the emission wavelengths is higher for the LiF film grown on Si substrate than for the LiF film grown on glass. An enhancement up to $100 \%$ is observed for the $F_{2}$ PLE band peaks in the investigated dose range, in agreement with previous investigations performed at a lower proton energy [21]. This value is in agreement with the results of the model applied to Si-LiF film bilayers [20], which estimate an enhancement between $90 \%$ and $150 \%$, as already observed in the PL spectra of Fig.4a. Again the main reason of this signal amplification is ascribed to the high reflectivity of silicon in the visible spectral range, where the absorption and emission bands of $\mathrm{F}_{2}$ and $\mathrm{F}_{3}{ }^{+} \mathrm{CCs}$ are located, although other complex effects due the presence of voids and grain boundaries in the polycrystalline matrix of the LiF films cannot be excluded.

\section{Conclusions}

Polycrystalline LiF thin films were grown by thermal evaporation on glass, fused silica(Suprasil ${ }^{\circledR}$ ) and $\operatorname{Si}(100)$ substrates in controlled conditions. Some physical parameters of the $\mathrm{LiF}$ films grown on fused silica substrate were determined starting from the measured specular reflectance and direct transmittance films spectra by using a best-fit procedure.

$\mathrm{LiF}$ films grown on glass and $\mathrm{Si}(100)$ substrates were irradiated by proton beams of nominal energy $27 \mathrm{MeV}$ at several doses in the range between $4.2 \times 10^{3}$ and $1.7 \times 10^{5}$. The investigation of PL and PLE spectra of radiation-induced $\mathrm{F}_{2}$ and $\mathrm{F}_{3}{ }^{+}$electronic defects shows a substrateenhancement in coloured LiF films grown on Si substrates with respect to LiF films deposited on glass in the same deposition run, at the investigated doses. This behaviour is mainly ascribed to the reflective properties of the Si substrate in the visible spectral range, where the absorption and emission bands of $\mathrm{F}_{2}$ and $\mathrm{F}_{3}{ }^{+} \mathrm{CCs}$ are located, although other complex effects due to the polycrystalline nature of the films cannot be excluded.

Further systematic studies are under way, in order to investigate the influence of the polycrystalline nature of thermally-evaporated LiF films on the formation efficiency of $\mathrm{F}_{2}$ and $\mathrm{F}_{3}{ }^{+}$electronic defects and to obtain higher substrate-enhanced photoluminescence intensities for improving the performance of the LiF film-based radiation detectors especially at lower doses.

\section{Acknowledgements}

This research was partly carried out within the TOP-IMPLART (Oncological Therapy with Protons - Intensity Modulated Proton Linear Accelerator for RadioTherapy) project, funded by Regione Lazio, Italy.

\section{References}

[1] R.M. Montereali, Point defects in thin insulating films of lithium fluoride for optical microsystems, in: Handbook of Thin Film Materials, Vol. 3 (Academic Press) 2002, pp. 399431. https://doi.org/10.1016/B978-012512908-4/50043-6

[2] S. W. S. McKeever, M. Moscovitch, P.D. Townsend, Thermoluminescence Dosimetry Materials: properties and uses, Nuclear Technology Publishing, Ashford, Kent TN23 1YW, England 1995. 
[3] J. Nahum, Optical Properties and Mechanism of Formation of Some F-aggregate enters in LiF, Phys. Rev. 158 (1967) 814. https://doi.org/10.1103/PhysRev.158.814

[4] G. Baldacchini, S. Bollanti, F. Bonfigli, F. Flora, P. Di Lazzaro, A. Lai, T. Marolo, R.M. Montereali, D. Murra, A. Faenov, T. Pikuz, E. Nichelatti, G. Tomassetti, A. Reale, L. Reale, A. Ritucci, T. Limongi, L. Palladino, M. Francucci, S. Martellucci, G. Petrocelli, A Novel Soft XRay Submicron Imaging Detector Based on Point Defects in LiF, Rev. Scient. Instr. 76, (2005) 113104-1. https://doi.org/10.1063/1.2130930

[5] F. Cosset, A. Celerier, B. Barelaud, J.C. Vareille, Thin reactive LiF films for nuclear sensors, Thin Solid Films 303 (1997) 191-195. https://doi.org/10.1016/S0040-6090(97)00070-9

[6] G. Baldacchini, F. Bonfigli, A. Faenov, F. Flora, R.M. Montereali, A. Pace, T. Pikuz, L. Reale, Lithium Fluoride as a Novel X-Ray Image Detector for Biological $\mu$-World Capture, J. Nanosci. Nanotechnol. 3 (6) (2003) 483-486. https://doi.org/10.1166/jnn.2003.023

[7] G. Tomassetti, A. Ritucci, A. Reale, L. Arizza, F. Flora, R.M. Montereali, A. Faenov, T. Pikuz, Two-Beam Interferometric Encoding of Photoluminescent Gratings in LiF Crystals by High-Brightness Tabletop Soft X-Ray Laser, Appl. Phys. Lett. 85 (18) (2004) 4163-4165. https://doi.org/10.1063/1.1812841

[8] M. Piccinini, F. Ambrosini, A. Ampollini, L. Picardi, C. Ronsivalle, F. Bonfigli, S. Libera, E. Nichelatti, M.A. Vincenti and R.M. Montereali, Photoluminescence of radiation-induced color centers in lithium fluoride thin films for advanced diagnostics of proton beams, Appl. Phys. Lett. 106 (2015) 261108. https://doi.org/10.1063/1.4923403

[9] M. Piccinini, E. Nichelatti, A. Ampollini, L. Picardi, C. Ronsivalle, F. Bonfigli, S. Libera, M.A. Vincenti and R.M. Montereali, Proton beam dose-mapping via color centers in LiF thinfilm detectors by fluorescence microscopy, EPL 117 (2017) 3704. https://doi.org/10.1209/02955075/117/37004

[10]C. Ronsivalle, M. Carpanese, C. Marino, G. Messina, L. Picardi, S. Sandri, E. Basile, B. Caccia, D. M. Castelluccio, E. Cisbani, S. Frullani, F. Ghio, V. Macellari, M. Benassi, M. D’Andrea, and L. Strigari, The TOP-IMPLART Project, Eur. Phys. J. Plus 126 (2011) 7. https://doi.org/10.1140/epjp/i2011-11068-X

[11]J. F. Ziegler, M. D. Ziegler, J. P. Biersac, Optical Properties and Mechanism of Formation of Some F-aggregate centers in LiF, Nucl. Instrum. Methods B 268 (2010) 1818.

[12]P.B. Barna, M. Adamik, Fondamental structure forming phenomena of polycrystalline films and the structure zone model, Thin Solid Films 317 (1996) 27-33. https://doi.org/10.1016/S00406090(97)00503-8

[13] M. Montecchi, R.M. Montereali, E. Nichelatti, Reflectance and transmittance of a slightly inhomogeneous film bounded by rough, unparallel interfaces, Thin Solid Films 396 (2001) 262273. https://doi.org/10.1016/S0040-6090(01)01253-6

[14] M. Montecchi, R.M. Montereali, E. Nichelatti, Erratum to Reflectance and transmittance of a slightly inhomogeneous film bounded by rough, unparallel interfaces, Thin Solid Films 396 (2001) 262-273. https://doi.org/10.1016/S0040-6090(01)01253-6

[15] E.D. Palik, Handbook of Optical Constants of Solids, Academic Press, San Diego, 1985. 
[16] G.A. Niklasson, C.G. Granqvist, O. Hunderi, Effective medium models for the optical properties of inhomogeneous materials, Appl. Opt. 20 (1981) 26-30.

https://doi.org/10.1364/AO.20.000026

[17] P. Patnaik, Handbook of Inorganic Chemicals, McGraw-Hill, New York, 2003.

[18] G. Baldacchini, E. De Nicola, R. M. Montereali, A. Scacco, V. Kalinov, Optical bands of $F_{2}$ and $\mathrm{F}_{3}{ }^{+}$centers in LiF, J. Phys. Chem. Solids 61 (2000) 21. https://doi.org/10.1016/S00223697(99)00236-X

[19] R.M. Montereali, F. Bonfigli, M.A. Vincenti and E. Nichelatti, Versatile lithium fluoride thin-film solid-state detectors for nanoscale radiation imaging, Il Nuovo Cimento C 36 (2) (2013) 35.

[20]E. Nichelatti, R.M. Montereali, Photoluminescence from a homogeneous volume source within optical multilayer: analytical formulas, J. Opt. Soc. Am. A 29 (2012) 303.

https://doi.org/10.1364/JOSAA.29.000303

[21]M. Leoncini, M.A. Vincenti, F. Bonfigli, S. Libera, E. Nichelatti, M. Piccinini, A. Ampollini, L. Picardi, C. Ronsivalle, A. Mancini, A. Rufoloni, R.M. Montereali, Optical investigation of radiation-induced color centers in lithium fluoride thin films for low-energy proton-beam detectors, Opt. Mat. 88 (2019) 580-585.

https://doi.org/10.1016/j.optmat.2018.12.031 